\title{
Evaluation of the Sensitivity of Inhomogeneous Magnetization Transfer (ihMT) MRI for Multiple Sclerosis
}

\author{
(D)E. Van Obberghen, (D). Mchinda, (D) A. le Troter, (D) V.H. Prevost, (DP. Viout, (D) M. Guye, (D) G. Varma, (DD.C. Alsop, (D).-P. Ranjeva,
}

(D). Pelletier, DO. Girard, and DG. Duhamel

\begin{abstract}
BACKGROUND AND PURPOSE: Inhomogeneous magnetization transfer is a new endogenous MR imaging contrast mechanism that has demonstrated high specificity for myelin. Here, we tested the hypothesis that inhomogeneous magnetization transfer is sensitive to pathology in a population of patients with relapsing-remitting MS in a way that both differs from and complements conventional magnetization transfer.
\end{abstract}

MATERIALS AND METHODS: Twenty-five patients with relapsing-remitting MS and 20 healthy volunteers were enrolled in a prospective MR imaging research study, whose protocol included anatomic imaging, standard magnetization transfer, and inhomogeneous magnetization transfer imaging. Magnetization transfer and inhomogeneous magnetization transfer ratios measured in normal-appearing brain tissue and in MS lesions of patients were compared with values measured in control subjects. The potential association of inhomogeneous magnetization transfer ratio variations with the clinical scores (Expanded Disability Status Scale) of patients was further evaluated.

RESULTS: The magnetization transfer ratio and inhomogeneous magnetization transfer ratio measured in the thalami and frontal, occipital, and temporal WM of patients with MS were lower compared with those of controls $(P<.05)$. The mean inhomogeneous magnetization transfer ratio measured in lesions was lower than that in normal-appearing WM $(P<.05)$. Significant $(P<.05)$ negative correlations were found between the clinical scores and inhomogeneous magnetization transfer ratio measured in normal-appearing WM structures. Weaker nonsignificant correlation trends were found for the magnetization transfer ratio.

CONCLUSIONS: The sensitivity of the inhomogeneous magnetization transfer technique for MS was highlighted by the reduction in the inhomogeneous magnetization transfer ratio in MS lesions and in normal-appearing WM of patients compared with controls. Stronger correlations with the Expanded Disability Status Scale score were obtained with the inhomogeneous magnetization transfer ratio compared with the standard magnetization transfer ratio, which may be explained by the higher specificity of inhomogeneous magnetization transfer for myelin.

ABBREVIATIONS: $C C=$ corpus callosum; EDSS = Expanded Disability Status Scale; $F W M=$ frontal WM; IC = internal capsule; ihMT = inhomogeneous magnetization transfer; ihMTR = inhomogeneous magnetization transfer ratio; MT = magnetization transfer; MTR = magnetization transfer ratio; OCCWM = occipital WM; RRMS = relapsing-remitting MS; TEMPWM = temporal WM

M ultiple sclerosis is a chronic inflammatory, demyelinating autoimmune disease of the central nervous system. Whereas edema, inflammation, and axonal loss are part of the

Received October 4, 2017; accepted after revision December 22.

From Aix-Marseille Université (E.V.O., S.M., A.I.T., V.H.P., P.V., M.G., J.-P.R., J.P., O.G., G.D.), Centre de Résonance Magnétique Biologique et Médicale (CRMBM), UMR 7339 Centre National de Recherche Scientifique (CNRS), Marseille, France; Department of Radiology (G.V., D.C.A.), Division of MR Research, Beth Israel Deaconess Medical Center, Harvard Medical School, Boston, Massachusetts; and Aix-Marseille University (J.P.), Assistance Publique des Hôpitaux de Marseille (APHM), Hôpital de La Timone, Pôle de Neurosciences Cliniques, Service de Neurologie, Marseille, France.

V.H.P. received support from A*MIDEX ANR (Agence Nationale de la Recherche, $n^{\circ}$ ANRI1-IDEX-0001-02), the Investissements d'Avenir program of the French Government. S.M received support from l'Association pour la recherche sur la sclérose en plaques (ARSEP), 2015 and l'institut pour la recherche sur la moelle épinière et l'encéphale (IRME), 2016. pathologic processes of MS, demyelination stands out as a major contributor to its pathogenesis. Hence, obtaining a precise in vivo assessment of myelin content is urgently needed, especially considering the recent advances in our understanding of the molecular and cellular mechanisms regulating de- and re-myelination and the emergence of new therapies to promote re-myelination. The ability of MR imaging to produce contrasts sensitive to different tissues, combined with its noninvasiveness, has propelled it to a technique of choice for in vivo imaging. Direct imaging of

Please address correspondence to Guillaume Duhamel, PhD, Aix-Marseille Université, Centre de Résonance Magnétique Biologique et Médicale, CRMBM-CEMEREM UMR 7339 CNRS, Faculté de Médecine, 27 Boulevard Jean Moulin, 13005 Marseille, France; e-mail: guillaume.duhamel@univ-amu.fr

- Indicates open access to non-subscribers at www.ajnr.org

http://dx.doi.org/10.3174/ajnr.A5563 


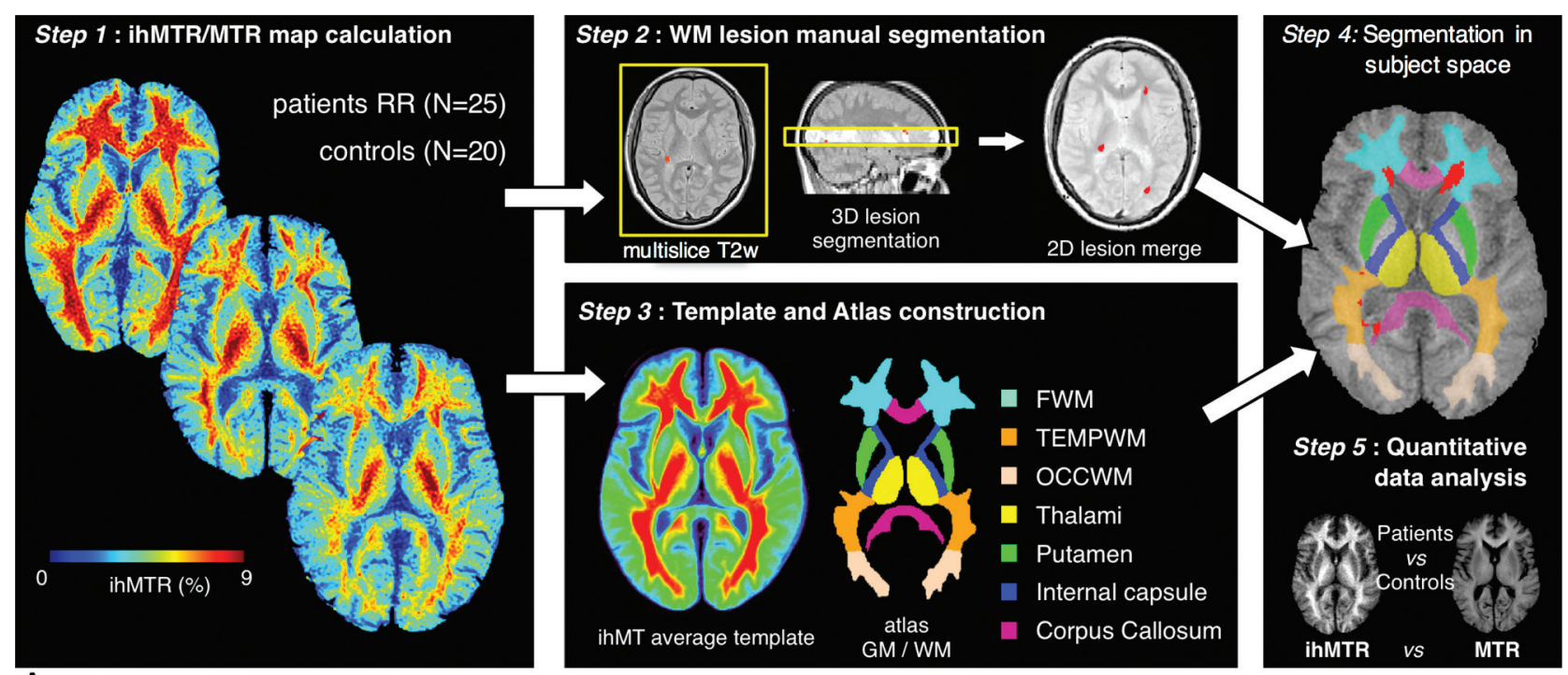

A

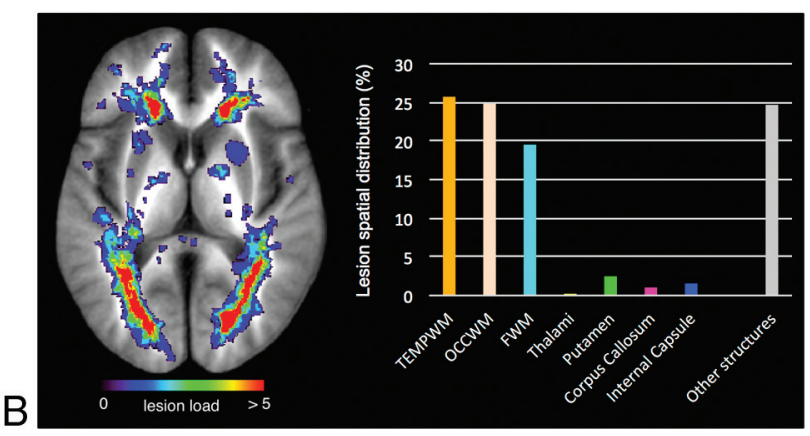

FIG 1. A, Postprocessing pipeline. Step 1: Quantitative MTR and ihMTR map calculation for controls and patients with RRMS. Step 2: Manual segmentation of WM lesions on the multislice $T_{2 W}$ images and reslicing to the single-slice $2 \mathrm{D}$ ihMT reference space. Step 3: Construction of the ihMT average template by realignment of the ihMT images of the control and RRMS subjects into a new common reference space. Construction of the ihMT atlas was based on the ihMT average template by manual segmentation of frontal, temporal, and occipital WM, internal capsule, corpus callosum, thalami, and putamen. Step 4: Automatic segmentation of individual ihMT images by projection of the ihMT atlas onto the individual native space of each control and patient. Individual lesion masks obtained in step 2 were removed from the ihMT atlas to generate normal-appearing tissue ROls. Step 5 : Quantitative measurement of ihMTR and MTR in the ROIs and lesions. B, Lesion-load map calculated over all the patients with RRMS and superimposed over the ihMT average template. The histogram shows the percentage of lesions within each brain structure.

myelin is, however, challenged by the MR imaging properties of the nonaqueous protons in the phospholipid bilayers that make up myelin. They produce a broad resonance spectrum spanning several kilohertz and leading to transverse relaxation times on the order of a few tens of microseconds, which correspond to a signal decay too fast to be detected directly. Advanced MR imaging techniques such as diffusion tensor imaging, myelin water fraction, ${ }^{1}$ and magnetization transfer $(\mathrm{MT})^{2,3}$ have thus been developed and their associated metrics have been shown to correlate, to some extent, with myelin content, thus providing an indirect assessment of myelin-related information.

The MT technique has been particularly used in clinical MS research studies, and its sensitivity for the pathology has been widely demonstrated. ${ }^{4-7}$ However, the MT signal is not specific to myelin, and other mechanisms involved in this complex disease (eg, inflammation, axonal loss) also contribute to the changes in MT metrics, thus preventing a complete understanding of myelin damage and repair in MS. The development of myelin-specific MR imaging techniques remains thus an active and highly focused area of research in which novel approaches are being sought. One promising example is the recently described inhomogeneous magnetization transfer (ihMT) technique, ${ }^{8}$ which can be envisioned as a method to image, in vivo, the dipolar order (characterized by a dipolar relaxation time constant) underlying broad tissue macromolecular lines. ${ }^{9-11}$ The long dipolar relaxation time values associated with myelinated structures compared with others $^{12,13}$ allow the ihMT technique to more selectively isolate the contribution of the myelin dipolar order within the broad MT signal, thereby providing higher specificity for myelinated structures. ${ }^{14}$ Studies performed on control subjects have shown exquisite contrast for CNS myelin-containing tissues, ${ }^{15,16}$ but the value of ihMT in clinical applications has yet to be demonstrated.

The current study aimed to provide preliminary evidence for the utility of ihMT in multiple sclerosis. We hypothesized that due to its strong specificity for myelin, ihMT should be sensitive to MS pathology and provide complementary information relative to the non-myelin-specific standard MT technique.

\section{MATERIALS AND METHODS \\ Subjects}

All subjects provided informed consent to participate in this prospective research study, which received the approval of the local 


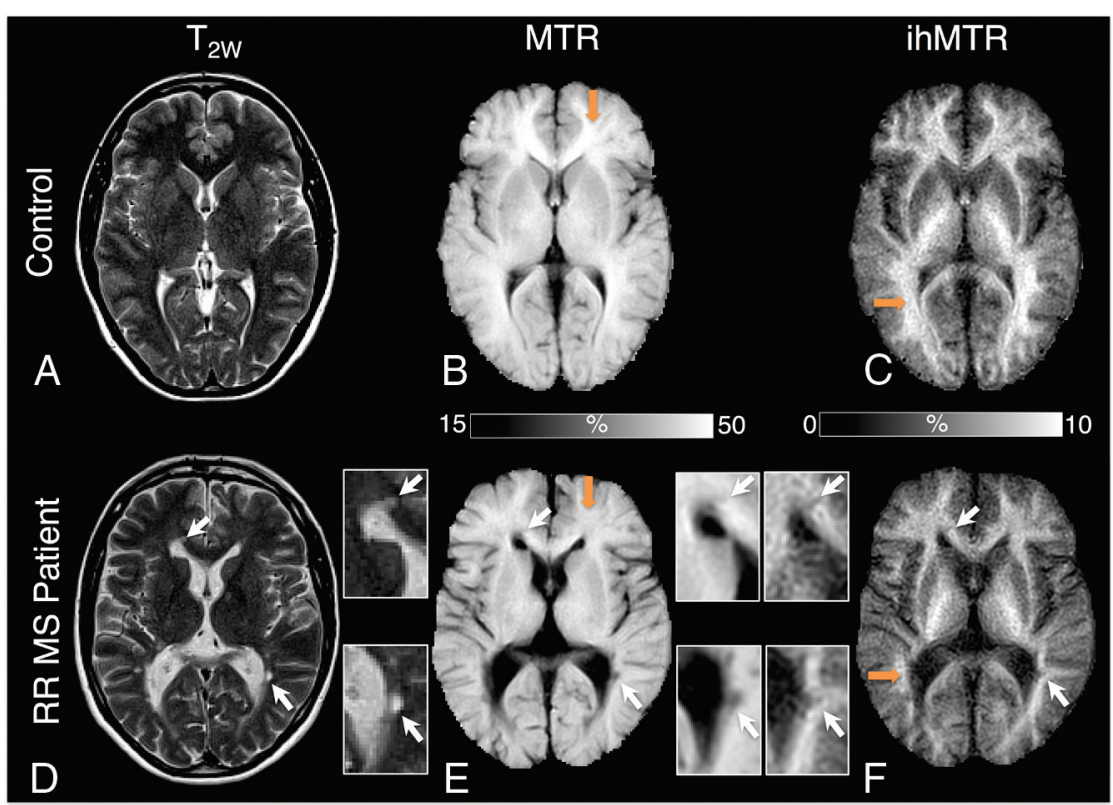

FIG 2. Representative anatomic $T_{2 W}$ images $(A$ and $D)$ and $M T R(B$ and $E$ ) and ihMTR ( $C$ and $F$ ) maps obtained on a control subject and a patient with RRMS after step 1 of the postprocessing procedure. Decrease of MTR and ihMTR signals is clear in patients ( $E$ and $F$ ) relative to controls ( $B$ and $C$ ) in the FWM and OCCWM (orange arrows). Brain areas encompassing lesions (white arrows) are shown in inserts for each technique. $T_{2 w}$ hyperintensities and MTR/ihMTR hypointensities are clearly visible in lesions.

research ethics committee (CPP Sud Méditerranée 1). Twentyfive patients with relapsing-remitting MS (RRMS) (20:5 women/ men; mean age, $42 / 41$ years; age range, $28-53$ years; mean disease duration, 11/9 years; disease duration range, $1-22$ years; $10 \mathrm{pa}-$ tients under disease-modifying treatment at the time of MR examinations; 9 patients with acute relapses at the time of MR imaging examinations) and 20 matched healthy volunteers (14:6 women/men; mean age, $42 / 39$ years; age range, $28-57$ years) were enrolled in this 1-year study. The exclusion criteria were alcohol or other drug abuse, history of psychiatric diseases, or any neurologic diseases other than MS. All patients underwent a standard neurologic examination by certified neurologists, and disability was assessed by the Kurtzke Expanded Disability Status Scale $(\text { EDSS })^{17}$ (mean group EDSS, 1.7; EDSS range, 0-6.5).

\section{MR Imaging Acquisition}

MR imaging examinations were performed on a 1.5T MR imaging system (Avanto; Siemens, Erlangen, Germany). The protocol included a 3D-FLAIR sequence $(\mathrm{TR} / \mathrm{TE} / \mathrm{TI}=6000 / 333 / 2200 \mathrm{~ms}$, $\mathrm{FOV}=256 \times 256 \times 176 \mathrm{~mm}^{3}$, matrix $=256 \times 256 \times 176$, isotropic spatial resolution $\left.=1 \mathrm{~mm}^{3}\right) ; \mathrm{a} \mathrm{T}_{2 \mathrm{~W}}$ multi-TE sequence $(\mathrm{TR}=2600$ $\mathrm{ms}, \mathrm{TEs}=14 / 85 \mathrm{~ms}, 44$ contiguous axial slices [3-mm thick] parallel to the anterior/posterior commissure line, FOV $=256 \times 256 \mathrm{~mm}^{2}$, matrix $=256 \times 256$, spatial resolution $\left.=1.0 \times 1.0 \times 3.0 \mathrm{~mm}^{3}\right)$; and an axial 2D-pulsed-ihMT HASTE sequence ${ }^{15}$ (circular polarized mode, $\mathrm{TR} / \mathrm{TE}=3000 / 21 \mathrm{~ms}, 789-\mathrm{Hz} /$ pixel readout bandwidth, echo spacing $=3.52 \mathrm{~ms}, 120^{\circ}$ spin-echo refocusing angle for limitation of the radiofrequency specific absorption rate, $\mathrm{FOV}=256 \times 256 \mathrm{~mm}^{2}$, matrix $=256 \times 256$, single slice of 9 -mm thick, spatial resolution $=$ $1.0 \times 1.0 \times 9.0 \mathrm{~mm}^{3}$ ). The slice was common to all subjects and chosen parallel to the anterior/posterior commissure line, midventricle to intercept the thalami. The ihMT preparation used the fol- lowing parameters, optimized for brain studies at $1.5 \mathrm{~T}^{15}$ : frequency-offset, $|\Delta f|=$ $7 \mathrm{kHz}$; pulse width/interpulse delay, $\mathrm{PW} /$ $\Delta \mathrm{t}=0.5 / 1 \mathrm{~ms}$; duration of saturation, $\tau=$ $700 \mathrm{~ms}$; intensity of saturation over the whole presaturation phase, $B_{1, \mathrm{RMS}}=7.0$ $\mu \mathrm{T}$. The $4 \mathrm{MT}$-prepared images required to generate the ihMT contrast (Equation 1) were averaged 20 times (20 NEX) corresponding to 4 minutes 15 seconds of acquisition time.

\section{Postprocessing}

The entire postprocessing procedure is summarized in Fig 1:

Step 1: Quantitative magnetization transfer ratio (MTR) and inhomogeneous magnetization transfer ratio (ihMTR) map calculation. Magnitude MT-weighted images derived from the ihMT sequence were processed using Matlab (Version R2012; MathWorks, Natick, Massachusetts) custom routines to generate, for all subjects (controls and patients), the composite ihMT images and the quantitative ihMT and MT ratios as defined by Girard et $\mathrm{al}^{15}$ and Prevost et $\mathrm{al}^{18}$ :

$$
\begin{gathered}
\text { ihMT }=\left(\mathrm{MT}^{+}+\mathrm{MT}^{-}-\mathrm{MT}^{+-}-\mathrm{MT}^{-+}\right), \\
\text {ihMTR }=\mathrm{ihMT} / \mathrm{S}_{0}, \\
\text { MTR }=1-\mathrm{MT}^{+} / \mathrm{S}_{0} .
\end{gathered}
$$

$\left(\mathrm{MT}^{+}, \mathrm{MT}^{-}\right)$and $\left(\mathrm{MT}^{+-}, \mathrm{MT}^{-+}\right)$correspond to MT-weighted images obtained with radiofrequency saturation at a single frequency offset $\left(+\Delta \mathrm{f}\right.$ or $-\Delta \mathrm{f}$, Fig $2 A$ of Girard et $\left.\mathrm{al}^{15}\right)$ and dual frequency offset $\left( \pm \Delta\right.$ f, Fig $2 B$ of Girard et $\mathrm{al}^{15}$ ), respectively. $S_{0}$ is the signal measured with the radiofrequency saturation power set to zero.

Step 2: Manual segmentation of WM lesions in the ihMT space. Following a procedure commonly used in clinical trials, segmentation of MS lesions (characterized by T2-hyperintensities) was performed on $\mathrm{T}_{2 \mathrm{~W}}$ images. For each patient, MS lesions were manually delineated on the 3 -mm-thick $\mathrm{T}_{2 \mathrm{~W}}$ images contained within the single 9-mm-thick ihMT image, using FSLView 3.2.0 (http://www.fmrib.ox.ac.uk/fsl/fslview). Note that FLAIR images were used as a secondary reference to improve the accuracy of the lesion location. The resulting 3D mask of lesions was resliced in the single-slice ihMT reference space, and voxels were considered lesions if at least 1 voxel was labeled as a lesion in the $\mathrm{T}_{2 \mathrm{~W}}$ images.

Step 3: ihMT template and atlas construction. ihMT images of all control subjects and patients were realigned into a new common reference space by a $2 \mathrm{D}$ nonlinear registration using a symmetric group-wise normalization procedure ${ }^{19,20}$ and further averaged to create a specific ihMT average template. Note that combining images of both patients and controls allowed optimization of the group-wise registration procedure by minimization 

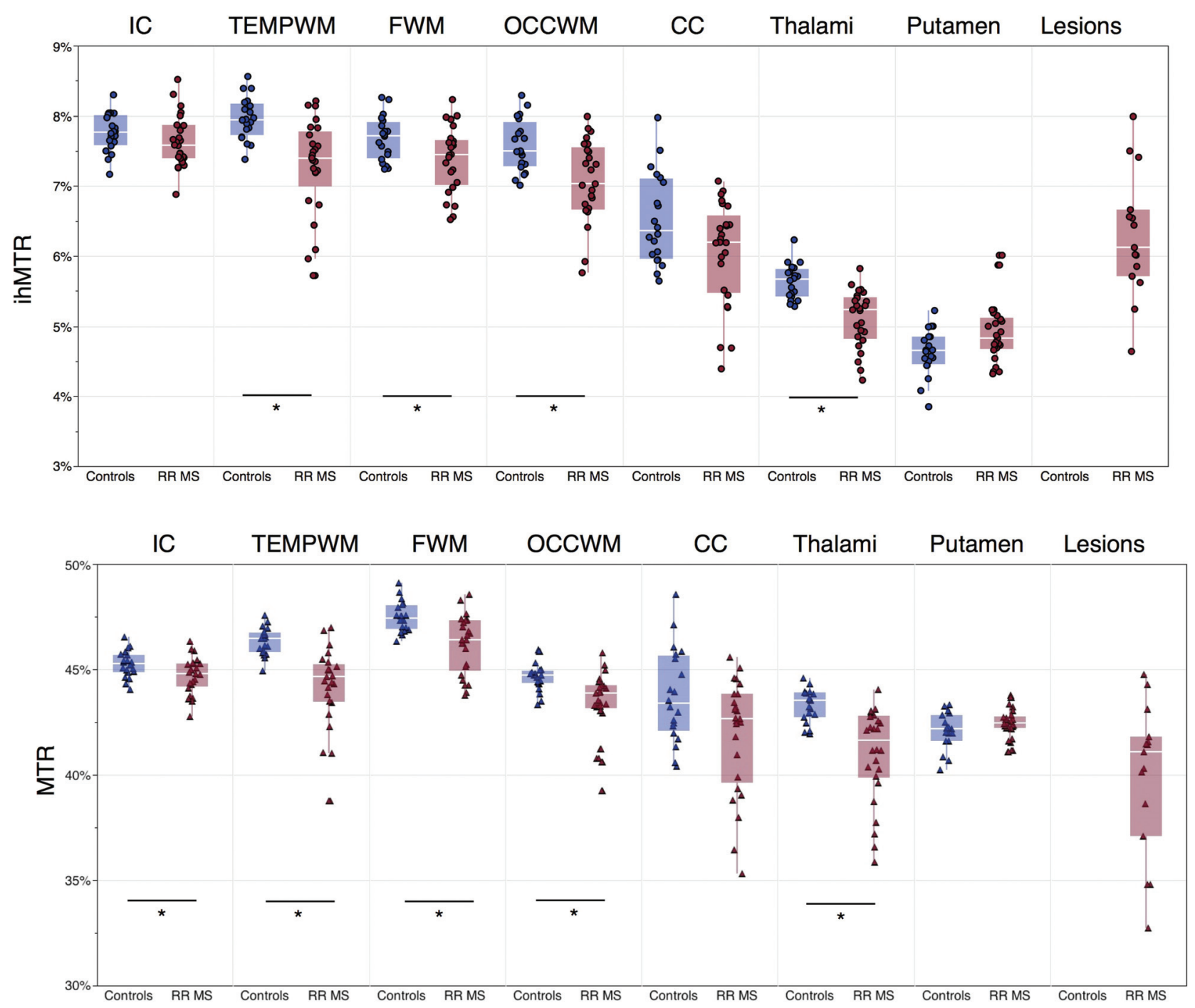

FIG 3. Mean MTR and ihMTR values measured in the 7 ROIs of the ihMT atlas (TEMPWM, FWM, OCCWM, CC, thalami, and putamen) for each control (blue markers) and patient with RRMS (pink markers). For patients, mean values measured in lesions are also reported. Boxplots indicate the median, upper and lower quartiles, and the minimum and maximum values excluding outliers. The asterisk indicates a significant difference $(P<.05)$ in values between controls and patients (comparison assessed by the nonparametric Wilcoxon rank test $[\alpha=.05]$ corrected by the Holm-Bonferroni method for multiple comparisons $[m=7$ brain structures]).

of image deformations from all individual subjects, especially the patients, to the common average template. Seven brain structures, including frontal, temporal, and occipital WM (FWM, TEMPWM, OCCWM), internal capsule (IC), corpus callosum (CC), thalami, and putamen were manually segmented on the ihMT average template by experienced clinical researchers (E.V.O. and J.P., with more than 10 years of experience in brain anatomy) using FSLView 3.2.0, to create a single 2D ihMT atlas. Note that partial volume effects induced by the large slice thickness of the ihMT images prevented accurate measurement in other finer brain structures.

Step 4: Segmentation in subject space. The ihMT atlas was automatically transformed into the individual native space of each subject (controls and patients) using inverse deformation fields estimated by the nonlinear process described in step 3. For each patient, the lesion mask derived in step 2 was removed from the ihMT atlas to generate normal-appearing tissue ROIs and enable calculation of normal-appearing tissue metrics.
Step 5: Quantitative data analysis. Mean ihMTR and MTR values were calculated in the 7 ROIs and in lesions.

\section{Statistics}

All statistical analyses were performed using JMP software (Version 9.0.1; SAS Institute, Cary, North Carolina). Three analyses were performed to identify diffuse and focal microstructural damage in patients with MS using ihMT and MT:

1) ihMTR and MTR values extracted from the 7 ROIs were compared between patients and controls using a nonparametric Wilcoxon rank test $(\alpha=.05)$ corrected for multiple comparisons ( $m=7$ ROIs) by the Holm-Bonferroni procedure.

2) ihMTR and MTR values measured in MS lesions were compared with values measured in the 7 normal-appearing tissue ROIs across all patients using a Kruskal-Wallis test corrected for multiple comparisons using the Steel-Dwass procedure. ${ }^{21}$

3) Computation and comparison of $z$ scores (a measure of how many SDs below or above the population mean a raw 
data value is) were used to assess the sensitivity of ihMT and MT to microstructural damage. The ihMTR and MTR $z$ scores of patients with RRMS relative to control subjects were calculated for each of the 7 ROIs according to the following equation:

Mean ihMTR and MTR values calculated in the 7 ROIs of the ihMT atlas and in lesions ${ }^{\mathrm{a}}$

\begin{tabular}{|c|c|c|c|c|}
\hline \multirow{2}{*}{$\begin{array}{c}\text { Brain } \\
\text { Structure }\end{array}$} & \multicolumn{2}{|c|}{ ihMTR (\%) } & \multicolumn{2}{|c|}{ MTR (\%) } \\
\hline & Controls & Patients & Controls & Patients \\
\hline \multirow[t]{2}{*}{ IC } & $7.8 \pm$ & & 0.6 & 44.7 \\
\hline & \multicolumn{2}{|c|}{$P=.16$} & \multicolumn{2}{|c|}{$P=.02^{b}$} \\
\hline TEMPWM & \multicolumn{2}{|c|}{$P<.001^{\mathrm{b}}$} & \multicolumn{2}{|c|}{$P<.001^{\mathrm{b}}$} \\
\hline FWM & \multicolumn{2}{|c|}{$P=.01^{\mathrm{b}}$} & \multicolumn{2}{|c|}{$P=.01^{\mathrm{b}}$} \\
\hline OCCWM & \multicolumn{2}{|c|}{$P=.004^{\mathrm{b}}$} & \multicolumn{2}{|c|}{$P=.005^{\mathrm{b}}$} \\
\hline CC & $\begin{array}{r}6.5 \pm 0.6 \\
P=\end{array}$ & $\begin{array}{l}6.0 \pm 0.7 \\
09\end{array}$ & $\begin{array}{r}43.7 \pm 2.2 \\
P\end{array}$ & $\begin{array}{l}41.9 \pm 2.7 \\
.07\end{array}$ \\
\hline Thalami & \multicolumn{2}{|c|}{$P<.001^{b}$} & \multicolumn{2}{|c|}{$P<.001^{\mathrm{b}}$} \\
\hline Putamen & \multicolumn{2}{|c|}{$P=.03$} & \multicolumn{2}{|c|}{$P=.09$} \\
\hline Lesions & NA & $6.3 \pm 0.9^{c}$ & NA & $39.9 \pm 3.6^{\circ}$ \\
\hline
\end{tabular}

Note:-NA indicates not applicable.

${ }^{a}$ ihMTR and MTR data are expressed as mean value \pm SD. Between-group comparison ( $P$ value): comparison of mean ihMTR and MTR between patients with RRMS and controls was assessed by the nonparametric Wilcoxon rank test $(\alpha=.05)$ corrected by the Holm-Bonferroni method for multiple comparisons ( $m=7$ brain structures). ${ }^{\mathrm{b}}$ Statistically significant.

'Within-patient group: ihMTR and MTR values in lesions statistically different (Kruskal-Wallis test corrected for multiple comparisons using the Steel-Dwass procedure) from values in all normal-appearing WM structures $(P<.05)$ except the corpus callosum $(P>.5)$.

\section{Z scores}

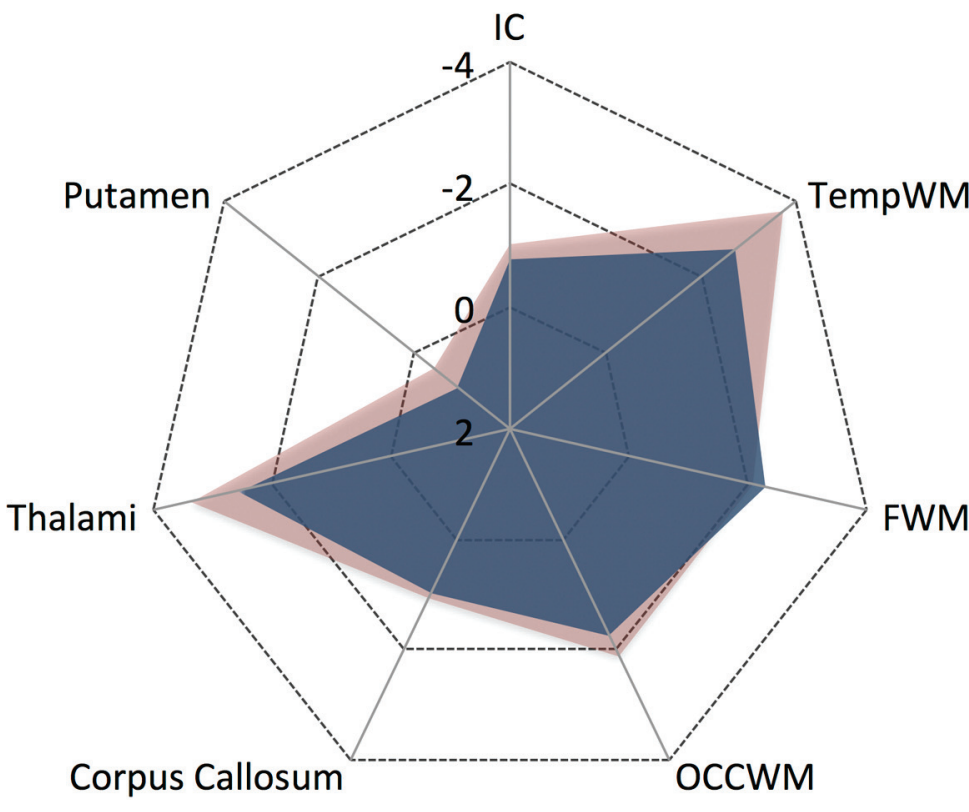

FIG 4. Radar plot of mean ihMTR and MTR $z$ scores of patients with RRMS relative to control subjects calculated for each brain structure of the ihMT atlas. A tendency (not significant, $P>$.1) for lower values was observed with MTR compared with ihMTR in the IC, TEMPWM, OCCWM, CC, and thalami.
MTR

ihMTR

$$
\mathrm{Z} \operatorname{Score}(X)_{\mathrm{ROI}}^{\text {patient,i }}=\left(\frac{X^{\text {patient,i }}-\mu^{\text {controls }}}{\sigma^{\text {controls }}}\right)_{\text {ROI }},
$$

where $X$ represents the value of ihMTR or MTR, $\mu^{\text {controls }}$ and $\sigma^{\text {controls }}$ represent the control group mean and SD of $X$ values. Mean ihMTR and MTR $z$ scores calculated over the patients with RRMS were compared using a nonparametric Wilcoxon rank test $(\alpha=.05)$.

Finally, the potential of ihMT to provide complementary information relative to standard MT was evaluated by the analysis of correlations between MTR and ihMTR values measured in the normal-appearing tissue ROIs with the EDSS clinical scores of patients with RRMS, using a statistical nonparametric Spearman rank test $(\alpha=.05)$ corrected for multiple comparison $(m=6$ ROIs) by the Holm-Bonferroni procedure.

\section{RESULTS}

Sensitivity of ihMT to Microstructural Damage in MS

On qualitative examination, the ihMTR and MTR maps of patients with MS exhibited focal hypointense areas in WM brain structures. This is illustrated in Fig 2 in a patient with reduced MTR and ihMTR in the FWM and OCCWM compared with a control subject. As shown in Fig 3 and in the Table, apart from the putamen, quantitative ihMTR and MTR values measured in patients with MS were lower compared with those of controls in the structures of the ihMT atlas (by trend in the IC and CC for ihMTR and in the CC for MTR; significant elsewhere). Additionally and despite pronounced partial volume effects, lesions (indicated by white arrows on Fig 2) could be identified on both ihMTR and MTR maps and exhibited hypointensities compared with the surrounding normal-appearing tissue. More generally, mean ihMTR and MTR values measured in lesions, which were distributed among the main WM structures of the ihMT atlas structures (Fig 1B), were lower than those in normal-appearing WM structures (not significant in the CC, $P=.7$; significant in the IC, TEMPWM, FWM, and OCCWM; $P<.05)$.

Mean patient ihMTR and MTR $z$ scores were close to or lower than -2 in WM structures (Fig 4). A tendency for lower $z$ score values was observed with MTR compared with ihMTR in normalappearing WM, but this was not significant $(P>.1)$.

\section{Correlation of ihMT with Clinical Disability}

Typical ihMTR and MTR maps of patients with MS with various values of EDSS are shown in Fig 5. Significant $(P \leq .05)$ negative correlations were found between the EDSS scores and ihMTR values measured in all normal-appearing WM structures (except OCCWM) and the thalami. Weaker, nonsignificant trends were found for MTR values (Fig 6). 


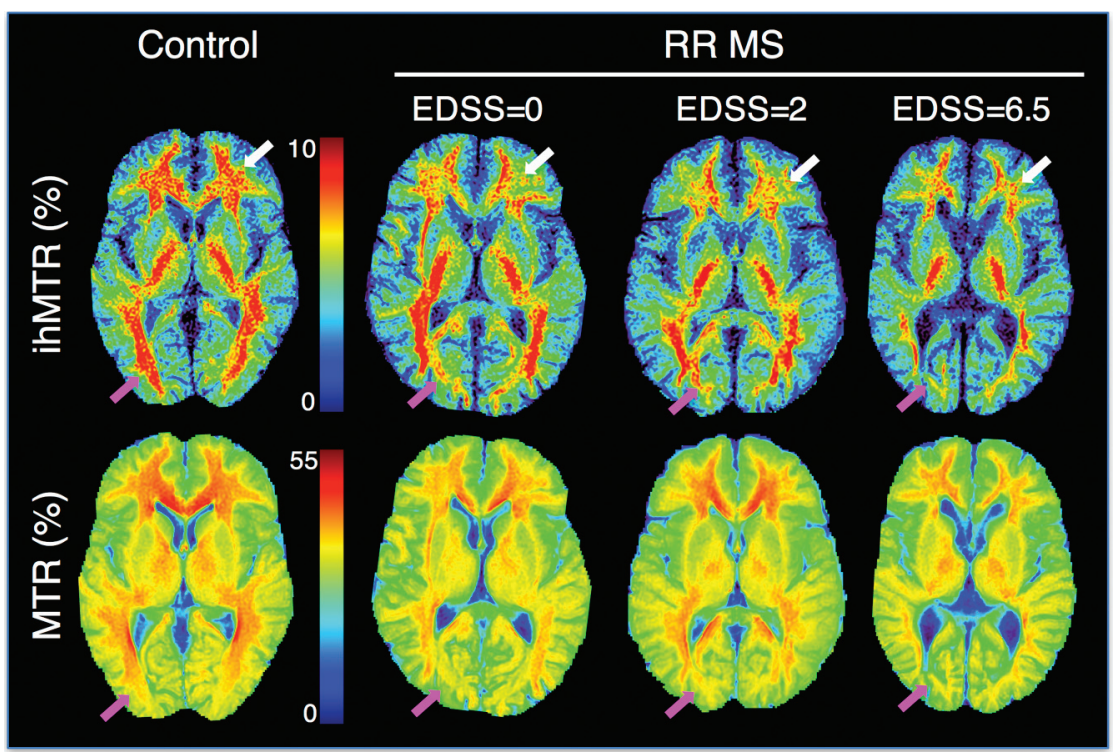

FIG 5. Representative ihMTR and MTR maps obtained after step 1 of the postprocessing procedure for a control subject and for patients with RRMS with various values of EDSS. Progressive loss of signal with increasing EDSS is noticed in occipital WM for both MTR and ihMTR (pink arrows). In frontal WM though, the decrease in values is more apparent with ihMTR (white arrows).

MTR vs. EDSS

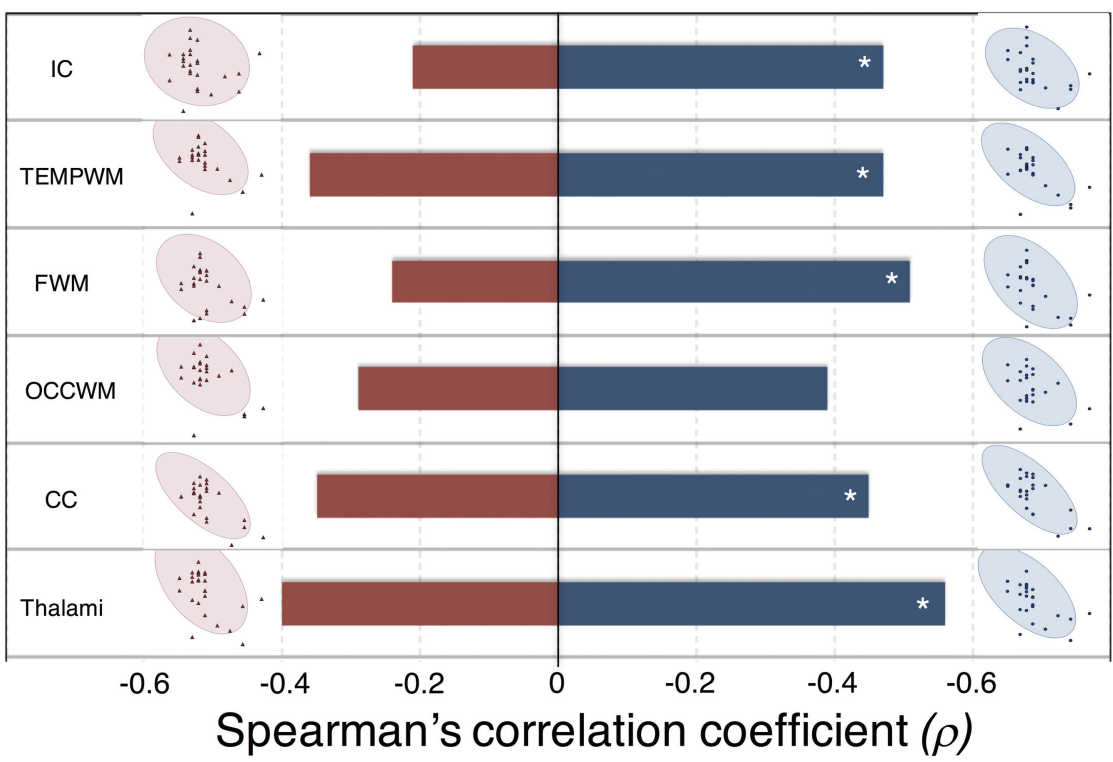

FIG 6. Correlation analysis of ihMTR versus EDSS scores of patients with RRMS. Mean MTR (pink markers) and ihMTR (blue markers) values measured in each structure of the ihMT atlas plotted as a function of the EDSS score and the density ellipses $(\alpha=.95)$ are shown. Bar plots indicate the Spearman correlation coefficient: The asterisk indicates statistically significant ( $\alpha=.05$, corrected by the Holm-Bonferroni method for multiple comparisons [ $m=6$ brain structures]). For ihMTR, significant negative correlations with EDSS were obtained in all structures (except the OCCWM); conversely, for the MTR, only a trend for low negative correlations with EDSS was obtained in all structures.

\section{DISCUSSION}

One of the key findings of this first report of ihMT in a clinical context is the significant reduction of ihMT signal in patients with multiple sclerosis compared with controls, hence demonstrating the sensitivity of this new technique for MS-related physiopathologic processes. Of additional interest, our results also highlighted a correlation of ihMT metrics with clinical disability, whereas MT metrics did not. This finding validates the hypothesis that ihMT and conventional MT provide complementary information. Combined, these results, albeit preliminary, support the use of ihMT in further clinical studies because its higher specificity for myelin could be advantageously used in the assessment of patients with MS.

In numerous studies, ${ }^{22}$ including the present report, conventional MT has been shown to be sensitive to tissue disorders in MS through changes in MTRs (lower MTR values in MS lesions, subtle significant diffuse decrease in MTR in normal-appearing tissue in patients). Similarly, the significant changes obtained in ihMTR values along with the ihMTR $z$ score intensities are key findings supporting the sensitivity of ihMT for the MS pathology.

The association between clinical disability and conventional MT is debatable. Whereas some studies have reported significant correlations between EDSS and MTR histogram features of normal-appearing brain tissue, ${ }^{23,24}$ others have suggested that the MTR of lesions in white matter, rather than in normal-appearing tissue, relates to the disability in patients with MS. ${ }^{4,25}$ Most important, correlations between MTR and clinical outcomes were predominantly detected for the highest EDSS $(>3)$ scores. ${ }^{25-27}$ Hence, the trend for a negative correlation of MTR in normalappearing tissue with the EDSS score (not significant) observed in our study is in agreement with these general findings. Overall, these controversial reports reflect confounding contributions to the standard MT signal induced by its sensitivity to multiple pathophysiologic mechanisms underlying $\mathrm{MS},{ }^{28}$ which limits its ability to relate clinical deficits to underlying regional pathology. Conversely, the potential of ihMT to derive metrics that more faithfully reflect the severity of the disease was apparent from the stronger significant correlations of ihMTR with EDSS. More generally, the lower sensitivity of ihMTR to the normal-appearing WM difference from controls but higher correlation with disability could suggest that ihMT is primarily detecting myelin changes that are more closely linked to disability, while MTR primarily detects inflammation-linked edema, one of the causes of normal-appearing WM changes in MS. ${ }^{28}$ Hence, the combined use of these 2 
contrasts and other myelin-sensitive modalities, such as diffusion MR imaging, in further studies may help disentangle the complex pathologic mechanisms occurring in MS.

Caution is advised, however, in drawing definitive conclusions because this preliminary study has some limitations. First, the cohort of patients was rather small and heterogeneous regarding disease duration and clinical status; these features may have mitigated the expected MTR and ihMTR variations. This relatively low number of patients may also explain why, though statistically significant, the correlations of ihMT and EDSS did not appear visually highly convincing. A stronger relationship between ihMTR variations and demyelination/remyelination processes might be established by focusing on the early onset of the disease following the detection of active lesions and by monitoring the ihMT parameters at different stages (inflammation, demyelination, remyelination, gliosis). Also, looking at potential correlations between ihMT metrics and clinical scores (eg, Multiple Sclerosis Functional Composite score) that assess other functional aspects of the disease than those assessed by EDSS should be considered in further studies to establish a more comprehensive relationship between ihMT- and MS-related impairment.

From a technical perspective, despite the care taken in the atlas construction, partial volume effects produced by the large slice thickness induced measurement bias, which may have led to underestimation of the variations of MTR and ihMTR in demyelinated lesions. Finally, the findings of this study could not be generalized at this stage because the single-slice ihMT approach did not allow whole-brain coverage. Hence, although the main objective of this study, demonstration of the sensitivity of ihMT for MS pathology, has been met, the technique must be improved to overcome these important technical limitations and make ihMT a practical tool applicable in daily clinical practice. In this context, the recently proposed sensitivity-enhanced 3D ihMT technique, which permits full brain coverage at an improved spatial resolution (1.5-mm isotropic) in 15 minutes $^{29}$ is very promising. Hence, combining 3D-ihMT with other imaging modalities (MT and DTI for inflammation and axonal loss evaluation) would be a useful tool for assessment of myelin impairment and for more accurate characterization of the complex MS disease mechanisms.

\section{CONCLUSIONS}

Results obtained in this preliminary investigation demonstrate the sensitivity of the ihMT technique for MS, characterized by significant reduction in ihMTR values in both demyelinated lesions and normal-appearing tissue of patients with RRMS. Stronger correlations with disability were obtained with ihMT compared with standard MT, which confirmed the complementarity of both techniques and may be explained by the higher specificity of ihMT for myelin. Our results warrant further exploration, which, if performed with the recently proposed 3D sensitivityenhanced ihMT approach, could make ihMT a propitious asset for in vivo assessment of myelin status in MS.

Disclosures: Samira Mchinda-RELATED: Grant: Institut pour la Recherche sur la Moelle épinière et l'Encéphale (IRME) 2016, Association pour la Recherche sur la Sclérose en Plaques (ARSEP) 2015.* David C. Alsop_UNRELATED: Grants/Grants Pending: GE Healthcare, Comments: I receive research support from GE Healthcare to advance perfusion imaging and magnetization transfer imaging methods*; Royal- ties: I receive postmarket royalties through my institution for licensing of inventions related to perfusion MRI. These technologies have been licensed by GE Healthcare, Philips Healthcare, Siemens, Hitachi Medical, and Animage Technology.* Jean Pelletier-UNRELATED: Board Membership: Biogen, Novartis, Merck, Roche, Teva Pharmaceutical Industries, MedDay*; Grants/Grants Pending: Biogen, Novartis, Merck, MedDay*; Travel/Accommodations/Meeting Expenses Unrelated to Activities Listed: Biogen, Novartis, Merck, Roche, Teva Pharmaceutical Industries, MedDay. Olivier Girard-RELATED: Grant: ARSEP 2015, Comments: supporting MRI costs*; UNRELATED: Employment: Aix-Marseille University, Comments: full-time research engineer; Grants/Grants Pending: ARSEP, Agence Nationale de la Recherche, SATT Sud Est*; Patents (Planned, Pending or Issued), Comments: patent pending, no license or financial interest up to now. Guillaume Duhamel—RELATED: Grant: IRME 2016, ARSEP 2015. *Money paid to institution.

\section{REFERENCES}

1. MacKay A, Whittall $\mathrm{K}$, Adler J, et al. In vivo visualization of myelin water in brain by magnetic resonance. Magn Reson Med 1994;31: 673-77 CrossRef Medline

2. Henkelman RM, Stanisz GJ, Graham SJ. Magnetization transfer in MRI: a review. NMR Biomed 2001;14:57-64 CrossRef Medline

3. Sled JG, Pike GB. Quantitative imaging of magnetization transfer exchange and relaxation properties in vivo using MRI. Magn Reson Med 2001;46:923-31 CrossRef Medline

4. Gass A, Barker GJ, Kidd D, et al. Correlation of magnetization transfer ratio with clinical disability in multiple sclerosis. Ann Neurol 1994;36:62-67 CrossRef Medline

5. Rocca MA, Mastronardo G, Rodegher M, et al. Long-term changes of magnetization transfer-derived measures from patients with relapsing-remitting and secondary progressive multiple sclerosis. AJNR Am J Neuroradiol 1999;20:821-27 Medline

6. Schmierer K, Scaravilli F, Altmann DR, et al. Magnetization transfer ratio and myelin in postmortem multiple sclerosis brain. Ann Neurol 2004;56:407-15 CrossRef Medline

7. Filippi M, Campi A, Dousset V, et al. A magnetization transfer imaging study of normal-appearing white matter in multiple sclerosis. Neurology 1995;45(3 Pt 1):478-82 CrossRef Medline

8. Varma G, Duhamel G, de Bazelaire C, et al. Magnetization transfer from inhomogeneously broadened lines: a potential marker for myelin. Magn Reson Med 2015;73:614-22 CrossRef Medline

9. Varma G, Girard OM, Prevost VH, et al. Interpretation of magnetization transfer from inhomogeneously broadened lines (ihMT) in tissues as a dipolar order effect within motion restricted molecules. J Magn Reson 2015;260:67-76 CrossRef Medline

10. Swanson SD, Malyarenko DI, Fabiilli ML, et al. Molecular, dynamic, and structural origin of inhomogeneous magnetization transfer in lipid membranes. Magn Reson Med 2017;77:1318-28 CrossRef Medline

11. Manning AP, Chang KL, MacKay AL, et al. The physical mechanism of "inhomogeneous" magnetization transfer MRI. J Magn Reson 2017;274:125-36 CrossRef Medline

12. Varma G, Girard OM, Prevost VH, et al. In vivo measurement of a new source of contrast, the dipolar relaxation time, $T_{1 \mathrm{D}}$, using a modified inhomogeneous magnetization transfer (ihMT) sequence. Magn Reson Med 2017;78:1362-72 CrossRef Medline

13. Prevost VH, Girard OM, Mchinda S, et al. Optimization of inhomogeneous magnetization transfer (ihMT) MRI contrast for preclinical studies using dipolar relaxation time $\left(T_{1 \mathrm{D}}\right)$ filtering. $N M R$ Biomed 2017;30:e3706 CrossRef

14. Prevost VH, Girard OM, Cayre M, et al. Validation of inhomogeneous magnetization transfer (ihMT) as a myelin biomarker. In: Proceedings of the Annual Meeting and Exhibition of the International Society for Magnetic Resonance in Medicine, Honolulu, Hawaii. April 22-24, 2017:1498

15. Girard OM, Prevost VH, Varma G, et al. Magnetization transfer from inhomogeneously broadened lines (ihMT): experimental optimization of saturation parameters for human brain imaging at $\mathbf{1 . 5}$ Tesla. Magn Reson Med 2015;73:2111-21 CrossRef Medline

16. Girard OM, Callot V, Prevost VH, et al. Magnetization transfer from inhomogeneously broadened lines (ihMT): improved imaging 
strategy for spinal cord applications. Magn Reson Med 2017;77: 581-91 CrossRef Medline

17. Kurtzke JF. Rating neurologic impairment in multiple sclerosis: an expanded disability status scale (EDSS). Neurology 1983;33:1444-52 CrossRef Medline

18. Prevost VH, Girard OM, Varma G, et al. Minimizing the effects of magnetization transfer asymmetry on inhomogeneous magnetization transfer (ihMT) at ultra-high magnetic field (11.75 T). MAGMA 2016; 29:699-709 CrossRef Medline

19. Avants BB, Tustison NJ, Song G, et al. A reproducible evaluation of ANTs similarity metric performance in brain image registration. Neuroimage 2011;54:2033-44 CrossRef Medline

20. Wu G, Jia H, Wang Q, et al. Emergence of groupwise registration in MR brain study. In: Liang $\mathrm{H}$, Bronzino JD, Peterson DR, eds. Biosignal Processing: Principles and Practices. Boca Raton, Florida: CRC Press; 2012: chap 5

21. Neuhäuser M, Bretz F. Nonparametric all-pairs multiple comparisons. Biom J 2001;43:571-80

22. Filippi M, Agosta F. Magnetization transfer MRI in multiple sclerosis. J Neuroimaging 2007;17:22S-26S. CrossRef Medline

23. Traboulsee A, Dehmeshki J, Peters KR, et al. Disability in multiple sclerosis is related to normal appearing brain tissue MTR histogram abnormalities. Mult Scler 2003;9:566-73 CrossRef Medline
24. Vrenken H, Pouwels PJ, Ropele S, et al. Magnetization transfer ratio measurement in multiple sclerosis normal-appearing brain tissue: limited differences with controls but relationships with clinical and MR measures of disease. Mult Scler 2007;13: 708-16 CrossRef Medline

25. Amann M, Papadopoulou A, Andelova M, et al. Magnetization transfer ratio in lesions rather than normal-appearing brain relates to disability in patients with multiple sclerosis. J Neurol 2015;262: 1909-17 CrossRef Medline

26. De Stefano N. Brain damage as detected by magnetization transfer imaging is less pronounced in benign than in early relapsing multiple sclerosis. Brain 2006;129:2008-16 CrossRef Medline

27. Santos AC, Narayanan S, de Stefano N, et al. Magnetization transfer can predict clinical evolution in patients with multiple sclerosis. J Neurol 2002;249:662-68 CrossRef Medline

28. Moll NM, Rietsch AM, Thomas S, et al. Multiple sclerosis normalappearing white matter: pathology-imaging correlations. Ann Neurol 2011;70:764-73 CrossRef Medline

29. Mchinda S, Varma G, Prevost VH, et al. Whole brain inhomogeneous magnetization transfer (ihMT) imaging: sensitivity enhancement within a steady-state gradient echo sequence. Magn Reson Med 2017 Sep 23. [Epub ahead of print] CrossRef Medline 